\title{
Denoising of Digital Images using Consolidation of Edges and Separable Wavelet Transform
}

\author{
Bhumika A. Neole \\ Assistant Professor \\ Department of Electronics and \\ Communication Engineering \\ Shri Ramdeobaba College of \\ Engineering and Management, \\ Nagpur-440013, India
}

\author{
Bhagyashree V. Lad \\ Assistant Professor \\ Department of Electronics \\ Engineering \\ Shri Ramdeobaba College of \\ Engineering and Management, \\ Nagpur-440013, India
}

\author{
K. M. Bhurchandi, PhD \\ Professor \\ Department of Electronics and \\ Communication Engineering \\ VNIT, Nagpur-440010, India
}

\begin{abstract}
Denoising is still a challenging area of research due to its commercial and technical applications. We present a novel approach to image denoising using edge profile detection and edge preservation in spatial domain in presence of zero mean additive Gaussian noise. A Noisy image is initially preprocessed using the proposed local edge profile detection and subsequent edge preserving filtering in spatial domain followed further by the modified threshold bivariate shrinkage algorithm. The proposed technique does not require any estimate of standard deviation of noise $(\sigma)$ present in the image. Performance of the proposed algorithm is presented in terms of PSNR and SSIM on a variety of test images containing a wide range of $\sigma$ starting from 15 to 100 . The performance of the proposed algorithm is better than NL means and Bivariate Shrinkage while it's comparable with BM3D.
\end{abstract}

\section{Keywords}

DWT, Local profile edge detection, Bivariate Shrinkage

\section{INTRODUCTION}

Image and video communication form a major portion of data acquired, interpreted, stored and transferred. Obviously all the information transmitted in the form of digital image or video need to have the best possible quality to represent the maximum possible information. But right from acquisition to transmission, the image or video information is often corrupted with noise of unknown characteristics and magnitude. The noise spectrum many times overlaps that of images or videos leading to loss of fine information during denoising. Thus removal of noise while preserving maximum possible details of image information without any knowledge of signal(image) or noise characteristics is a challenging problem faced by researchers. This challenge is accepted by many researchers and various good denoising algorithms have been proposed like NL means, Bivariate Shrinkage, BM3D etc. [1],[2],[3],[4],[5]. This paper reports a novel attempt to improve the performance of Bivariate Shrinkage in presence of a wide range of standard deviation of noise on a wide variety of images. If an image is $I(x, y)$ and noise is $N(x, y)$ then a noisy image $Y(x, y)$ is modeled as;

$$
Y(x, y)=I(x, y)+N(x, y)
$$

where, $I$ and $N$ are considered highly uncorrelated. As characteristics of $I(x, y)$ and $N(x, y)$ are unknown,Image denoising takes shape of an inverse problem that aims to minimize the noise $N(x, y)$, so that $Y(x, y)$ is denoised.

Complete removal of noise is not possible, but it can be minimized using some techniques on noisy images. Many
Denoising algorithms have been proposed for different types of noise, each has its own advantages and limitations. Here we present a new image denoising algorithm that uses spatial domain edge preservation and Discrete Wavelet Transform based Bivariate shrinkage algorithm for denoising [3]. At a high noise density, detail information like edges in the original image is distorted by noise. Thus, it is required to recover the closest approximation of the original image by minimizing noise and preserving the fine and detail information content.

Preserving the edges and reconstructing image objects with smooth surfaces have been a challenge for a long time [4]. Wavelet transform preserves both, the time domain and frequency domain information and also the relation between them. Wavelet theory has so far been widely used for edge detection [2], denoising [5], signal processing using hidden Markov models[8], image enhancement [6], pattern classification [9], pattern recognition [10]. The success of Wavelet transform was mainly due to its good performance in both the dimensions at varying resolutions; but one at a time [8] due to its orthogonality. Since Wavelet transform in two dimensions are obtained by a tensor product of one dimensional wavelets, it is good at isolating discontinuities across horizontal or vertical edges.

Wavelet Local Denoise Reconstruction Algorithm based on non-separable Multi Resolution Analysis (MRA) was presented by Jin Guoying and Chen Hongye [3]. In this work, the projected data of a noisy image is decomposed by two level wavelet decomposition and approximated \& detailed coefficients are obtained. The coefficients are then denoised using soft thresholding technique and then the denoised image was reconstructed using inverse wavelet transform. In nonseparable MRA, whole image is processed simultaneously and not along rows and columns. It simultaneously yields a multidirectional sensitive decomposition. However the nonseparable MRA techniques are computationally complex. V.NagaPrudhvi Raj and T Venkateswarlu proposed denoising of medical Images using Undecimated Wavelet Transform [4]. Many of the wavelet based denoising algorithms use DWT (Discrete Wavelet Transform) in the decomposition stage which suffers from shift variance and boundary discontinuity[24],[29-31]. This problem is eliminated to a large extent using Undecimated Wavelet Transform[34-38]. The problems of resolution were satisfactorily addressed by multi-resolution and multi-scale capabilities of Wavelet transform [5],[6] to large extent.

G.Y. Chen and B. Kegl [11-12],[32] incorporated dual tree complex Wavelet into Ridgelet transform and enhanced its directional sensitivity to improve its denoising performance 
using thresholding of Ridgelet coefficients. Since noise usually consists of high frequency content, thresholding or partial truncating of details coefficients, followed by reconstruction, leads to suppression of overall noise, at the cost of some distortion. Many techniques using wavelet-based thresholding have been reported in literature [6-7],[9], [13],[17],[21].

Edge Detection of SAR Images have been implemented using Shift-Invariant DWT and Binarization Method proposed by WangCan et al. [22-23]. This yields a fast unsupervised noiserobustness edge detection method in synthetic aperture radar (SAR) images. Input image is first decomposed with shiftinvariant discrete wavelet transform (DWT) and edge enhancement is achieved using the subbands of the SAR images. A fast unsupervised hybrid method of the binarization and edge detection is used for edge detection in SAR images. This incorporates speckle reduction and edge detection as a single process so that complex operations are avoided.

P.R. Hill et al. presented an image denoising method based on dual tree statistical models for complex wavelet transform [7]. The Dual-Tree Complex Wavelet Transform (DT-CWT) along with bivariate analysis techniques that condition the shrinkages on spatially related coefficients across neighbouring scales (DWT levels). These comparatively recent techniques have denoised the real and imaginary components of the DT-CWT coefficients separately, followed by the inverse transform. Transform domain denoising techniques have achieved good results over the last few decades, preservation of fine spatial domain information is also utmost importance. Thus hybrid techniques, which process noisy images in both; spatial as well as transform domain are very important. Hard thresholding of transform coefficients is used along with the multiscale decompositions. Wavelet shrinkage denoising can be achieved using "soft thresholding" and many algorithms have been developed based on this approach. Another important method has been presented by A. Buades and et al. [14-16] for image denoising. This method named NL means, computes a normalized and weighted mean of Gaussians to represent contribution of neighboring pixels for each pixel of a noisy image. It uses an exponential kernel and Euclidean distances of all the neighboring pixels from the pixel to converge to a weight matrix for each pixel.

Noise is usually removed by thresholding transform coefficients in transform domain or by some type of weighted averaging (filtering) in spatial domain. Wavelet transform or its derivatives are so far most popular for denoising applications. But wavelet transform doesn't capture all the properties of images though is computationally simple. So in this paper, a new technique that first reconstructs the distorted edges and smoothens regions in spatial domain as a part of preprocessing is proposed. Further wavelet transform based bivariate shrinkage algorithm with the proposed and modified threshold is used for denoising. The proposed algorithm yields good denoising performance while preserving the fine details and sharp edges at low computational overhead. The results endorse edge preserving and smoothening capabilities of the proposed approach and denoising abilities of Discrete Wavelet Transform using improved threshold Bivariate Shrinkage.

Section 1 has introduced the denoising state of the art and the proposed work. We present Bivariate Shrinkage algorithm using Discrete wavelet transform in section 2. Section 3 presents the proposed edge profile detection and preservation preprocessing along with the modified threshold bivariate shrinkage. Section IV presents performance, benchmarking and discussions on the proposed algorithm. Section V concludes on the work.

\section{TRANSFORM BASED TECHNIQUES}

In this section, we initially present noise variance estimation from the available noisy image itself using the Dohono and Johnstone [18]. In the proposed work, we use this variance for further denoising using bivariate shrinkage algorithm.

\subsection{Noise Variance Estimation using Robust Median Estimator}

The thresholding technique used here requires an estimate of the noise level $\sigma$ that is locally computed using the finest level detail DWT coefficients. The usual standard deviation of the local noisy pixel values is not a good estimator, unless the function is reasonably flat which is a most unlikely situation in practical images [18]. Thus estimation of $\sigma$ in wavelet domain using wavelet coefficients at the finest resolution level is an empirical solution. The reason for considering only the finest level is that the corresponding empirical wavelet coefficients tend to consist mostly noise. Since there is some signal present even at this level, a proposed a robust estimate of the noise level $\sigma$ using the $n^{\text {th }}$ level DWT coefficients $W j$ based on the median absolute deviation given by,

$\sigma_{n^{2}}=\frac{\text { median }\left\{\left|W_{j}\right|: j=1,2,3, \ldots \ldots, k\right\}}{0.6745}$

Here $\mathrm{W}_{0}, W_{1} \ldots \ldots$ etc are the detail coefficients at the finest level.

\subsection{Bivariate Shrinkage}

Neighbouring DWT coefficients are strongly dependent on each other. There is also a strong dependency between a coefficient, its parent (adjacent coarser scale locations), and their siblings (adjacent spatial locations). In the proposed work, we considering the dependencies only between a coefficient and its parent and sibling are expected to contain more noise components than its children. In [19], it is suggested that the pdf of a DWT coefficient band is Gaussian. A linear Bayesian estimator is proposed for the estimation of neighbouring coefficients as in [20],[25-28]. The Bayesian estimation problem is modified so as to take into account the statistical dependency between a coefficient and its parent [33].

A bivariate shrinkage rule is described using a DWT coefficient and its parent. Bayes rule allows us to write the estimation in terms of the probability densities of noise and the prior density of the wavelet coefficients. In order to use this equation to estimate the original signal, we must know both pdfs i.e. a coefficient and its parent. We assume the noise is Gaussian, and the separable 2D noise probability density function $p_{n}(n)$ defined on the two successive DWT levels' coefficients ( 1 and 2) of DWT is given as,

$p_{n}(n)=\frac{1}{2 \pi \sigma_{n^{2}}} \cdot \exp \left(-\frac{n_{1}^{2}+n_{2}^{2}}{2 \sigma_{n^{2}}}\right)$

where, $n_{1}$ and $n_{2}$ are noise samples at the successive DWT levels.

Sunder and Selesnick [20] proposed four empirical models on the non-non noisy DWT coefficients and their standard 
deviation, each with its own advantages and disadvantages. From those four models the first model is given by:

$p_{w}(w)=\frac{3}{2 \pi \sigma_{n^{2}}} \cdot \exp \left(-\frac{\sqrt{3}}{\sigma} \sqrt{w_{1}^{2}+w_{2}^{2}}\right)$

With this pdf, $w_{1}$ and $w_{2}$ are uncorrelated but not independent The standard deviation $\sigma$ is computed over a $3 \times 3$ neighborhood. Let $w_{2}$ represents the parent of $w_{1}$, where $w_{2}$ is the wavelet coefficient at the same level as $w_{1}$, but at the next coarser scale. $y_{1}$ and $y_{2}$ are noisy observations of $w_{1}$ and $w_{2}$ respectively which are given by

$y_{1}=w_{1}+n_{1}$

$y_{2}=w_{2}+n_{2}$

The standard MAP estimator for $\mathrm{w}$ given the corrupted observation y is

$\widehat{w}(y)=\operatorname{argmax} p_{w \mid y}(w \mid y)$

From above equation, according to Bayes rules we can obtain probability densities of noise and the prior density of the wavelet coefficients as shown in equation (3) and (4) .

MAP estimator is found corresponding to the model given in equation (4) and $f(w)=\log p_{w}(w)$ can be defined as

$f(w)=\log \left(\frac{3}{2 \pi \sigma_{n^{2}}}\right)-\frac{\sqrt{3}}{\sigma} \sqrt{w_{1}^{2}+w_{2}^{2}}$

From [20], the derivatives of $f(w)$ w.r.t. $w_{1}$ and $w_{2}$ are given respectively computed as $f_{1}$ and $f_{2}$ in equation (9) and (10).

$f_{1}(w)=-\frac{\sqrt{3} w_{1}}{\sigma \sqrt{w_{1}^{2}+w_{2}^{2}}}$
$f_{2}(w)=-\frac{\sqrt{3} w_{2}}{\sigma \sqrt{w_{1}^{2}+w_{2}^{2}}}$

Solving (3), (4), (7) and (8) using (9) and (10), the MAP estimator (or "the joint shrinkage function") is written as in (11),

$\widehat{W_{1}}=\frac{\left(\sqrt{y_{1}^{2}+y_{2}^{2}}-\frac{\sqrt{3} \sigma n^{2}}{\sigma}\right)_{+}}{\sqrt{y_{1}^{2}+y_{2}^{2}}} \cdot y_{1}$

where, the threshold $T$ for estimating the wavelet coefficients is given as,

$T=\frac{\sqrt{3} \sigma_{n}^{2}}{\sigma_{s}}$

\section{PROPOSED EDGE STRENGTHENING BIVARIATE SHRINKAGE}

In this section, we present a preprocessing and post processing edge strengthening algorithm using Local Profile Edge detection along with the modified bivariate shrinkage algorithm based on the modified probability model and the threshold. It may be noted that the proposed algorithm does not require any noise standard deviation value as an input. Rather, it is estimated based on the local DWT coefficient values across the levels unlike Non-local means, Bivariate Shrinkage and BM3D algorithm. Here we presented the modified model, by changing the 2D noise model presented in (4) as below in equation (13) $p_{w}(w)=\frac{5}{2 \pi \sigma^{2}} \cdot \exp \left(-\frac{\sqrt{5}}{\sigma} \sqrt{w_{1}^{2}+w_{2}^{2}}\right)$

Based on (13), we propose a MAP modified estimator of $\widehat{w_{1}}$ as discussed in section 2. Here we have modified the equation for $w_{1}$ by considering the calculation over $5 \times 5$ neighborhood as in (13) and the proposed denoising algorithm uses this model and the modified threshold.

So the modified Wavelet Coefficient for implementing a Bivariate shrinkage for estimating $\widehat{w_{1}}$ is given by;

$\widehat{W_{1}}=\frac{\left(\sqrt{y_{1}^{2}+y_{2}^{2}}-\frac{\sqrt{5} \sigma n^{2}}{\sigma}\right)_{+}}{\sqrt{y_{1}^{2}+y_{2}^{2}}} \cdot y_{1}$

Here ' + ' indicates only positive values of the (.) in (14); the negative values of (.) will be considered 0 and accordingly the estimate of $\widehat{w_{1}}$ is also 0 . In scalar soft thresholding, for all coefficients, the threshold value is fixed and independent on other coefficients, if the coefficient is below the threshold value, we make it zero. It is clear that the estimated value depends on the parent value.The smaller the parent value, the greater the shrinkage and better is the denoising. Thus, the estimated value of $\widehat{W_{1}}$ with the new threshold for estimating the wavelet coefficients is as in (14) and the modified threshold is given by (15). However, further increase in the shrinkage i.e. $T$ due to the increased neighbourhood sizes results in blurring.

$T=\frac{\sqrt{5} \sigma_{n}^{2}}{\sigma_{s}}$

Thus, in the proposed work, the Bivariate Shrinkage is implemented with the wavelet coefficients using the modified threshold.

Wavelet Denoising using Bivariate Shrinkage improves the denoising performance of an image. But while denoising using DWT bivariate shrinkage the sharp edge details of an image are not preserved. Edges are significant local changes in the image intensities and are important features for preserving visual quality of images. Edges typically occur on boundaries between two different regions in an image. Edge detection is frequently the first step in recovering information from images. Due to its importance, edge detection still continues to be an active research area.

\subsection{Spatial Local Profile Edge Detection}

The proposed local profile edge detection algorithm is presented in this section. A local neighbourhood $3 \times 3$ tile is used to detect an edge at its center position in spatial domain. This helps in reconstructing the edges, distorted due to the effect of noise before application of the modified threshold bivariate shrinkage and also after it. It has been observed that a denoising algorithm like Bivariate Shrinkage blurs edges in noisy images. Here we hypothesize that if there are already blurred edges in the original image then they are slightly blurred further in the process of denoising but the effect may not be noted very easily. However if sharp edges are blurred, it may considerably affect the visual quality of the original images. Thus if a local edge and its gradient direction in spatial domain are precisely detected before applying the denoising technique, the edge can be protected using a customized denoising process for the specific edge type. Thus, the proposed local profile edge detection precisely achieves the preliminary denoising while protecting image edges. There are many classical edge detection methods available. The edges of an image can be detected directly by processing 
in the spatial domain or in transform domain. In spatial domain also, many edge detecting techniques like Sobel edge detector, Prewitt edge detector and so on already exist but are computationally complex. Moreover they require some type of threshold. In this section, we propose a simple and novel local edge and its direction detection algorithm to detect edges of an image in the spatial domain.

We propose that total 24 types of edges pass through a $3 \times 3$ local neighbourhood tile center. We classify the image edges in the $3 \times 3$ tiles in the 24 types in addition to non-edge (smooth) tile. Each edge type is assigned a number. This vector together is called local edge profile. Using this Local edge Profile detection algorithm we detect vertical, horizontal, rising and falling (oblique) edges of total 24 different types and a smooth tile. The local edge profile is detected all over the image. Thus we can have local edge profile of each noisy image in terms of mean and edge number. The edge profile at each position is used to find the similar or dissimilar tiles. And according to the detected edge number and its direction we process the tile by averaging parallel to the edge direction so as to preserve the local edge and minimize the noise. Algorithm for the Local Profile Edge Detection is given as below;

\section{Algorithm 1:}

1. For each overlapping $3 \times 3$ tile of noise corrupted image check for the edge type present i.e. horizontal, vertical, diagonal rising or falling edge as below.

2. Calculate the potential sum of the shaded area as summation of the intensities parallel to the shown edge.

If the difference of shaded area is greater than the threshold computed below from the tile itself, then an edge is present. Calculate the threshold $t$ by the given formula:

$t=\operatorname{mode}(\delta)$

where $\delta$ is the difference vector of every pixel in the $3 \times 3$ tile from the center pixel.

3. Calculate the difference between the edge potentials. If it is more than $3 x$ standard deviation of the tile in the specific direction, the edge direction is available in the tile. Replace the respective pixel intensities parallel to the edge by the potential divided by three.

4. Average all such nine estimates of the overlapping tile.

5. Replace each pixel of $3 \times 3$ tile by the mean of that tile if there is no edge is detected.

6. Thus, we obtain the denoised image using local profile edge detection algorithm.

Table 1 presents the detailed description of the no edge and possible different edges in a3x3 tile, in figure 1 that shows light edges on dark background represented by edge number 2 to 13. Similarly there can be twelve dark edges on light background represented by edge no 13 to 26 .

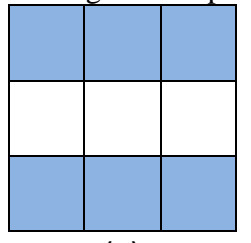

(a)

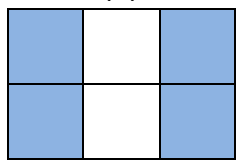

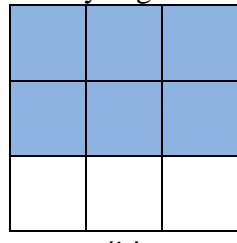

(b)

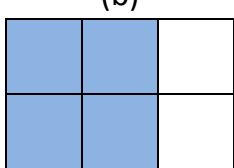

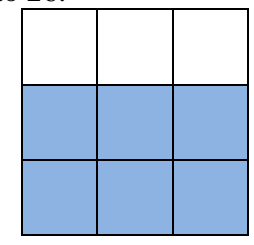

(c) (d)

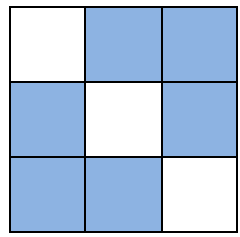

(g)

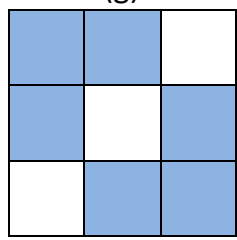

(j)

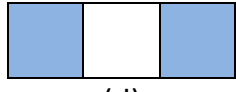

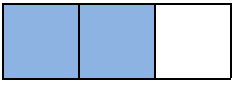

(e)

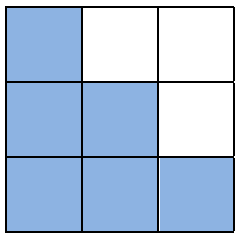

(h)

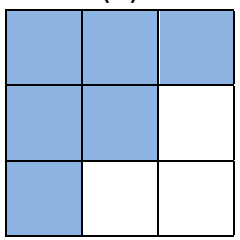

(k)

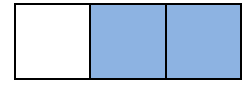

(f)

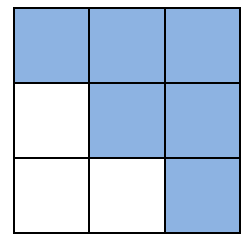

(i)

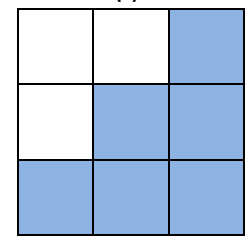

(I)
Fig 1: Different types of edges (a)-(c) Horizontal $\left(0^{\circ}\right.$ edges ,(d)-(f) Vertical edges $\left(90^{\circ}\right.$ edges $)$, (g)-(l)Rising $\left(+45^{\circ}\right)$ and Falling $\left(-45^{\circ}\right)$ edges.

Thus total twenty four types of edges can be identified most probably in each $3 \times 3$ tile. Also there can be smooth or no edge tiles represented by edge number 1 and 14 . The labels 'above' and 'below' indicate the position of the edge with respect to center of the tile. The label 'center' indicates an edge passing through center of the tile. 'Light' indicates light edge on dark back ground and 'Dark' indicates dark edge on light background. More number of dark pixels in a tile indicate dark back ground while more number of the light pixels indicate light background. An edge is supposed to have intensity opposite to the background. It should be noted that the $3 \times 3$ tile is converted into a binary representation for such edge classification. This can be easily done by finding the mean of a $3 \times 3$ tile $(\mu)$ and bi-thresholding the tile intensities using the mean. Every tile provides its edge number available w.r.t. the center of the tile.

Once the edge number available at the center pixel of the tile is detected the tile is processes by averaging the dark and light original image intensities parallel to the edge orientations and substituting the respective dark and light pixel means at the respective pixel positions. Thus for the non-boundary pixels for $3 \times 3$ size overlapping tiles, each pixel will have nine estimates due to the nine overlapping tile at each pixel position. Average of these nine at each pixel position provides the final pixel intensity for edge consolidation.

\subsection{Modified Threshold Bivariate shrinkage}

Using the local edge profile detection, processing and the modified bivariate shrinkage function, an effective and low complexity locally adaptive image denoising algorithm is developed. This shrinkage function requires the prior knowledge of the noise variance and the signal variance for each wavelet coefficient as mentioned in section 2 . Therefore the bivariate shrinkage algorithm requires computation of the following:

1. DWT coefficients till $6^{\text {th }}$ level.

2. The local noise variance using robust median estimator on the detailed $6^{\text {th }}$ level coefficients.

3. Modified DWT coefficients using the threshold in (15).

4. Compute inverse DWT of the sixth level DWT. 
Table 1: Edge types in a 3x3 tile

\begin{tabular}{|c|c|c|c|c|}
\hline $\begin{array}{l}\text { Eg. } \\
\text { No. }\end{array}$ & $\begin{array}{c}\text { Fig. } \\
1 \\
\end{array}$ & $\begin{array}{l}\text { Edge description } \\
\text { Dark background }\end{array}$ & $\begin{array}{l}\text { Eg. } \\
\text { No. }\end{array}$ & $\begin{array}{l}\text { Edge Description } \\
\text { Light background }\end{array}$ \\
\hline 1 & & Dark, no edge tile & 14 & Light no edge tile \\
\hline 2 & $\mathrm{a}$ & $\begin{array}{l}\text { Light, single pixel } \\
\text { thick, center, at } 0^{\circ}\end{array}$ & 15 & $\begin{array}{l}\text { Dark single pixel thick at } 0^{\circ} \\
\text { i.e. complement of Fig } 1(\text { a) }\end{array}$ \\
\hline 3 & $\mathrm{~b}$ & $\begin{array}{l}\text { Light, single pixel } \\
\text { thick, below, at } 0^{\circ}\end{array}$ & 16 & $\begin{array}{l}\text { Dark single pixel thick below at } \\
0^{\circ} \text { i.e. complement of Fig. } 1(\mathrm{~b})\end{array}$ \\
\hline 4 & $\mathrm{c}$ & $\begin{array}{l}\text { Light, single pixel } \\
\text { thick, above, at } 0^{\circ}\end{array}$ & 17 & $\begin{array}{l}\text { Dark, single pixel thick above at } \\
0^{\circ} \text { i.e. complement of Fig. } 1 \text { (c) }\end{array}$ \\
\hline 5 & d & $\begin{array}{l}\text { Light, single pixel } \\
\text { thick, center, at } 90^{\circ}\end{array}$ & 18 & $\begin{array}{l}\text { Dark, single pixel thick, center, } \\
\text { at } 90^{\circ} \text { i.e. complement of } \\
\text { Fig.1(d) }\end{array}$ \\
\hline 6 & $\mathrm{e}$ & $\begin{array}{l}\text { Light, single pixel } \\
\text { thick, right, at } 90^{\circ}\end{array}$ & 19 & $\begin{array}{l}\text { Dark, single pixel thick, right, at } \\
90^{\circ} \text {, i.e. complement of Fig.1(e) }\end{array}$ \\
\hline 7 & f & $\begin{array}{l}\text { Light, single pixel } \\
\text { thick, left, at } 90^{\circ}\end{array}$ & 20 & $\begin{array}{l}\text { Dark, single pixel thick, left, at } \\
90^{\circ} \text {, i.e. complement of Fig.1(f) }\end{array}$ \\
\hline 8 & $\mathrm{~g}$ & $\begin{array}{l}\text { Light, single pixel } \\
\text { thick,center,at } 135^{\circ}\end{array}$ & 21 & $\begin{array}{l}\text { Dark, single pixel thick, center, } \\
\text { at } 135^{\circ} \text {, i.e. complement of } \\
\text { Fig. } 1(\mathrm{~g})\end{array}$ \\
\hline 9 & h & $\begin{array}{l}\text { Light, } 2 \text { pixel thick, } \\
\text { above, at } 135^{\circ}\end{array}$ & 22 & $\begin{array}{l}\text { Dark, } 2 \text { pixel thick, above, at } \\
135^{\circ} \text {, i.e. complement of Fig. } 1(\mathrm{~h})\end{array}$ \\
\hline 10 & $\mathrm{i}$ & $\begin{array}{l}\text { Light, } 2 \text { pixel thick, } \\
\text { below, at } 135^{\circ}\end{array}$ & 23 & $\begin{array}{l}\text { Dark, } 2 \text { pixel thick, below, at } \\
135^{\circ} \text {, i.e. complement of Fig.1(i) }\end{array}$ \\
\hline 11 & $\mathrm{j}$ & $\begin{array}{l}\text { Light, single pixel } \\
\text { thick, center, at } 45^{\circ}\end{array}$ & 24 & $\begin{array}{l}\text { Dark, single pixel thick, center, } \\
\text { at } 45^{\circ} \text {, i.e. complement of } \\
\text { Fig.1(j) }\end{array}$ \\
\hline 12 & $\mathrm{k}$ & $\begin{array}{l}\text { Light, } 2 \text { pixel thick, } \\
\text { below, at } 45^{\circ}\end{array}$ & 25 & $\begin{array}{l}\text { Dark, } 2 \text { pixel thick, below, at } 45^{\circ} \\
\text { i.e. complement of Fig. } 1(\mathrm{k})\end{array}$ \\
\hline 13 & 1 & $\begin{array}{l}\text { Light, } 2 \text { pixel thick, } \\
\text { above, } 45^{\circ}\end{array}$ & 26 & $\begin{array}{l}\text { Dark, 2pixel thick, above, at } 45^{\circ} \\
\text { i.e. complement of Fig.1(1) }\end{array}$ \\
\hline
\end{tabular}

\subsection{Proposed Algorithm}

This section presents the proposed Edge preserving bivariate shrinkage denoising algorithm. As already said the proposed algorithm is a cascade of local profile edge detection and processing as a preprocessing technique followed by the modified bivariate shrinkage and again local profile edge detection and processing as a post-processing technique. Thus, the proposed algorithm sandwiches the modified bivariate shrinkage between the pre and post processing using local edge profile detection.

It is clear that, using $3 \times 3$ neighbourhood, we obtain 9 estimates of each pixel value of a noisy image during pre and post processing. These 9 estimates are obtained by shifting a $3 \times 3$ tile of an image along the columns and then along the rows by one pixel resulting in overlapped tiles. The final estimate at each pixel is achieved by averaging these nine estimates if the tile edge profile matches all over the image. This results in consolidation of the edges in the noisy image. As already described the noise distorts edges; this preprocessing consolidates the edge. Also a denoising algorithm smoothens the fine image details; so the post processing step again consolidates the edges to provide the final denoised image. Here the steps of proposed algorithm are presented below as Algorithm 2 .

\section{Algorithm 2:}

1. On the given noisy image a preprocessing edge strengthening algorithm Algorithm 1 is applied as a pre-proscessing step.

2. Compute Modified Bivariate Shrinkage on noisy image using the following process.

- Select window size $5 \times 5$ for signal variance computation and 6 numbers of levels for DWT computations.

- Compute DWT.
- Compute noise variance using (2) for each subband.

- Process each subband using (3) to yield denoised wavelet coefficient estimates at all the scales.

- Estimate the signal variance and threshold using (14) for each band for the selected window size.

- Estimate the denoised wavelet coefficients at all the levels using (14) and the threshold using (15).

- Compute inverse DWT to yield original image.

3. Also apply the Denoising algorithm using the local profile edge detection algorithm as explained in Section 3.2 to the output image of step 2 .

Thus, we obtain the resultant image after computing postprocessing edge strengthening algorithm alongwith the modified bivariate shrinkage algorithm.

\section{EXPERIMENTAL RESULT}

Experiments have been carried out on many natural images along with test images. The standard images are considered zero noise or reference images. Zero mean Gaussian noise of standard deviation from 15 to 100 has been added with all the images to yield the respective denoised images. The proposed algorithm was applied on the noisy images one by one and the respective denoised images and the corresponding PSNR and SSIM values have been computed for benchmarking. Though the experimentation has been performed on many images, a few representative results on the standard images have been presented in this section. It has been observed that the proposed technique yields the denoising performance comparable to the state of the art published denoising algorithms visually and quantitatively both. However, this performance can be considered better because, the other algorithms like BM3D and NL means require the noise standard deviation value as input to the algorithm. In the absence of this input or inaccurate input their performance considerably reduces. However, the proposed algorithm does not require any such noise parameter as input and thus it is completely automatic or blind. Even in the absence of this noise input parameter, the proposed algorithm yield matching performance with BM3D and much better performance compared to NL means. Thus the proposed algorithm can be considered fully automatic. The proposed algorithm also clearly outperforms the original Bivariate shrinkage algorithm. As already said, performance of the proposed algorithm is compared visually using denoised images and quantitatively using PSNR and SSIM. However it should be noted that, in the absence of the noise standard deviation estimate input, the denoising performance of the proposed algorithm falls considerably. As far as visual performance is considered, the edge preservation of the proposed algorithm is slightly better than BM3D while its much better than NL means.

Experiments for selecting the best neighbourhood have been carried out on tile sizes $3 \times 3,5 \times 5,7 \times 7$ and $9 \times 9$ on five standard images and the PSNR of denoising has been plotted against the noisy image tile size as in fig 2 . It has can be observed in figure 2 that we got consistently better PSNR results on all the images with the $5 \times 5$ tile size. Further increased tile size results in blurring of the images leading to reduction in PSNR. Thus for further experiments we use the fixed tile size of $5 \times 5$ on all the images. This is also consistent with our newly selected Bivariate shrinkage model in Eq. (13). It should be noted here that we have experimented with only even square tile sizes. It is possible to locate centre. of the tile if the tile size is odd; resulting in equal effect of the surrounding pixels on the centre pixel during processing. 


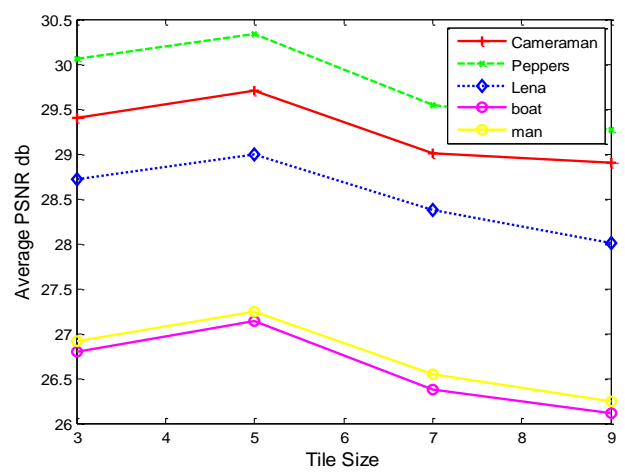

Fig 2: PSNR against the tile size for calculating noise $\sigma$

Results on five standard test gray images namely; cameraman, peppers, lena, boat and man, all of spatial resolution $512 \times 512$ pixels have been presented in Fig.2. This algorithm was tested at noise levels $15,25,35,50,80$ and 100. It is compared with NL means, BM3D and Bivariate Shrinkage. Performance analysis is done using the PSNR and SSIM measures in fig.4. Each PSNR value in the table is averaged. In this graph, the highest PSNR value among all is seen and yielded consistently. From this graph it is evident that using our modified bivariate shrinkage function with proposed algorithm provides better performance than using it with original empirical bivariate shrinkage.

Figure 3(a) and (b) show the PSNR against Standard deviation of input noise in Peppers and Lena images respectively. It also shows the results of the proposed algorithm on the Lena images corrupted by the Gaussian noise of 15 and 35 standard deviation. The result on the test images is shown in table 1. It can be seen that the results of proposed algorithm are comparable to other denoising techniques. Also it gives improved denoising in terms of PSNR and SSIM while preserving image edges. It has been verified that denoising performance of the proposed technique drops below NLmeans for a tile size of $3 \times 3$ but it is much improved for a tile size $5 \times 5$ for computation of the estimate of local standard deviation. Thus the proposed denoising techniques offers better performance for moderate Gaussian noise with standard deviation 15 to 35 at much lower computational cost compared to BM3D as shown in table2.

It is observed from results that on an average we are getting 12 dB PSNR improvement compared to noisy images. Fig. 4 presents the performance of the published algorithms along with the proposed algorithm in terms of PSNR on the two test images Lena and Peppers.

In fig. 5 visual results of the other algorithms along with the proposed algorithms are also presented. It can be seen that the noisy images' quality is degraded due to Gaussian noise of 15 and 35 standard deviation.

After applying denoising algorithm, denoising has been achieved to a large extent by all the algorithms. We can see that the denoising has been achieved by the proposed algorithm much better compared to NLmeans. The Nl-means algorithm introduces a lot of blurring due to the excessive averaging implemented as part of the algorithm. If we compare the results in terms of SSIM, it also shows huge improvement compared to the noisy images. The performance of the proposed algorithm is again comparable to the BM3D algorithm and much better than NL-means algorithm.

As the standard deviation of the noise increased, the performance of the proposed algorithm had an edge over that of NL means. The proposed algorithm the edges are protected to a large extent compared to even Baudes NL means and BM3D. As a result, even we are getting higher SSIM value as compared to Baudes NL means and closest to BM3D algorithm.

Table 3 presents the de-noising performance of the proposed algorithm along with Baudes NL means, Bivariate Shrinkage and BM3D methods on color images of Lena and Peppers. Zero mean Gaussian noise with standard deviation values of $15,25,35$ and 50 have been introduced in the original color images of Peppers and Lena of size $512 \times 512$. Here also we observe that there is an improvement in the PSNR and SSIM values of proposed algorithm compared to PSNR and SSIM values obtained by Baudes NL means. From table 2 and table 3 , it can be observed that the results of the proposed algorithm are comparable to the results of BM3D and are much better than the Baudes NL means algorithm and the conventional Bivariate shrinkage algorithm. It should also be noted that the proposed algorithm outperforms all other algorithms in terms of SSIM at high noise standard deviation.

Table 2: Benchmarking of the results obtained by Proposed Algorithm (Gray Images)

\begin{tabular}{|c|c|c|c|c|c|c|c|c|c|c|c|}
\hline \multirow[t]{2}{*}{ Image } & \multirow{2}{*}{$\frac{\text { SD }}{\sigma}$} & \multicolumn{2}{|c|}{$\begin{array}{l}\text { Noisy } \\
\text { Image }\end{array}$} & \multicolumn{2}{|c|}{\begin{tabular}{|c|}
$\begin{array}{c}\text { Buades NL } \\
\text { Means }\end{array}$ \\
\end{tabular}} & \multicolumn{2}{|c|}{$\begin{array}{c}\text { Bivariate } \\
\text { Shrinkage } \\
\end{array}$} & \multicolumn{2}{|c|}{ BM3D } & \multicolumn{2}{|c|}{$\begin{array}{c}\text { Proposed } \\
\text { Algorithm } \\
\end{array}$} \\
\hline & & PSNR & SSIM & PSNR & SSIM & PSNR & SSIM & PSNR & SSIM & PSNR & SSIM \\
\hline \multirow{6}{*}{$\begin{array}{c}\text { Camera } \\
\text { man }\end{array}$} & 15 & 24.6 & 0.72 & 32.99 & 0.91 & 34.4 & 0.93 & 35.76 & 0.95 & 35.32 & 0.93 \\
\hline & 25 & 20.2 & 0.54 & 30.52 & 0.86 & 31.5 & 0.88 & 33.18 & 0.92 & 32.45 & 0.90 \\
\hline & 35 & 17.2 & 0.43 & 28.8 & 0.83 & 29.6 & 0.83 & 31.52 & 0.90 & 30.69 & 0.86 \\
\hline & 50 & 14.7 & 0.34 & 26.63 & 0.78 & 27.10 & 0.73 & 29.94 & 0.86 & 28.63 & 0.79 \\
\hline & 80 & 11.4 & 0.20 & 23.82 & 0.66 & 24.51 & 0.65 & 27.74 & 0.81 & 26.21 & 0.70 \\
\hline & 100 & 10.1 & 0.15 & 22.67 & 0.60 & 23.21 & 0.61 & 26.62 & 0.78 & 25.01 & 0.65 \\
\hline \multirow{6}{*}{ Peppers } & 15 & 24.6 & 0.76 & 34.34 & 0.93 & 34.8 & 0.94 & 36.42 & 0.95 & 36.25 & 0.96 \\
\hline & 25 & 20.2 & 0.58 & 31.57 & 0.90 & 32.0 & 0.90 & 33.91 & 0.92 & 33.66 & 0.92 \\
\hline & 35 & 17.2 & 0.46 & 29.44 & 0.87 & 30.2 & 0.87 & 32.16 & 0.90 & 31.68 & 0.89 \\
\hline & 50 & 14.7 & 0.34 & 27.17 & 0.83 & 27.32 & 0.78 & 30.47 & 0.87 & 28.94 & 0.82 \\
\hline & 80 & 11.4 & 0.20 & 24.28 & 0.74 & 24.67 & 0.69 & 27.98 & 0.82 & 26.38 & 0.74 \\
\hline & 100 & 10.1 & 0.15 & 23.26 & 0.69 & 23.12 & 0.64 & 26.77 & 0.78 & 25.10 & 0.69 \\
\hline \multirow{6}{*}{ Lena } & 15 & 24.6 & 0.77 & 32.51 & 0.92 & 32.5 & 0.93 & 34.3 & 0.95 & 33.74 & 0.94 \\
\hline & 25 & 20.2 & 0.59 & 29.84 & 0.88 & 30.2 & 0.89 & 32.07 & 0.92 & 31.59 & 0.90 \\
\hline & 35 & 17.2 & 0.47 & 28.20 & 0.84 & 28.7 & 0.85 & 30.6 & 0.89 & 29.84 & 0.87 \\
\hline & 50 & 14.6 & 0.35 & 26.45 & 0.79 & 26.41 & 0.77 & 29.03 & 0.86 & 28.02 & 0.81 \\
\hline & 80 & 11.4 & 0.22 & 24.38 & 0.69 & 24.32 & 0.66 & 26.94 & 0.80 & 25.86 & 0.72 \\
\hline & 100 & 10.1 & 0.16 & 23.46 & 0.64 & 23.11 & 0.61 & 25.94 & 0.76 & 24.80 & 0.67 \\
\hline
\end{tabular}

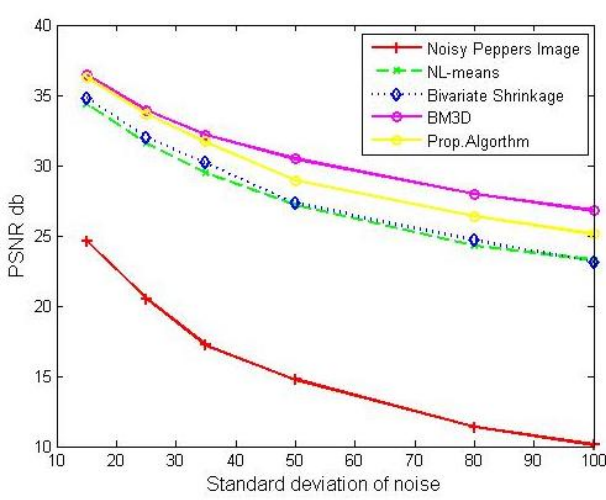

Fig 3(a): PSNR Vs Standard deviation of noise of Peppers Image 


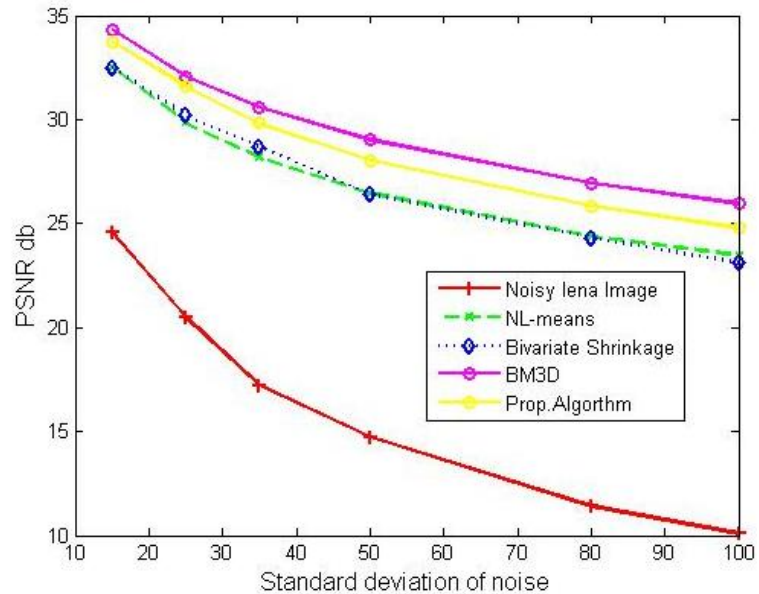

Fig 3(b): PSNR Vs Standard deviation of noise of Lena Image

Table 3: Benchmarking of the results obtained by Proposed Algorithm (Color Images)

\begin{tabular}{|c|c|c|c|c|c|c|c|c|c|c|c|}
\hline \multirow[t]{2}{*}{ Image } & \multirow{2}{*}{$\frac{\text { SD }}{\sigma}$} & \multicolumn{2}{|c|}{$\begin{array}{l}\text { Noisy } \\
\text { Image }\end{array}$} & \multicolumn{2}{|c|}{$\begin{array}{c}\text { Buades NL } \\
\text { Means }\end{array}$} & \multicolumn{2}{|c|}{$\begin{array}{c}\text { Bivariate } \\
\text { Shrinkage }\end{array}$} & \multicolumn{2}{|c|}{ BM3D } & \multicolumn{2}{|c|}{$\begin{array}{r}\text { Proposed } \\
\text { Algorithm }\end{array}$} \\
\hline & & PSNR & SSIM & PSNR & SSIM & PSNR & SSIM & PSNR & SSIM & PSNR & SSIM \\
\hline \multirow{4}{*}{ Lena } & 15 & 24.6 & 0.76 & 32.30 & 0.92 & 31.58 & 0.90 & 33.92 & 0.95 & 33.47 & 0.93 \\
\hline & 25 & 20.48 & 0.59 & 29.84 & 0.88 & 29.59 & 0.88 & 32.27 & 0.93 & 31.60 & 0.91 \\
\hline & 35 & 17.2 & 0.43 & 28.20 & 0.84 & 28.27 & 0.87 & 30.89 & 0.90 & 29.94 & 0.89 \\
\hline & 50 & 15.02 & 0.34 & 26.45 & 0.77 & 26.81 & 0.86 & 29.81 & 0.89 & 28.76 & 0.87 \\
\hline \multirow{4}{*}{$\begin{array}{c}\text { Peppe } \\
\text { rs }\end{array}$} & 15 & 24.68 & 0.76 & 31.92 & 0.93 & 31.55 & 0.91 & 32.67 & 0.94 & 32.18 & 0.92 \\
\hline & 25 & 20.37 & 0.58 & 29.90 & 0.89 & 29.88 & 0.89 & 31.13 & 0.92 & 30.61 & 0.91 \\
\hline & 35 & 17.60 & 0.46 & 28.33 & 0.85 & 28.72 & 0.86 & 29.94 & 0.89 & 29.39 & 0.87 \\
\hline & 50 & 14.81 & 0.34 & 26.52 & 0.80 & 27.68 & 0.82 & 28.92 & 0.87 & 28.12 & 0.83 \\
\hline
\end{tabular}

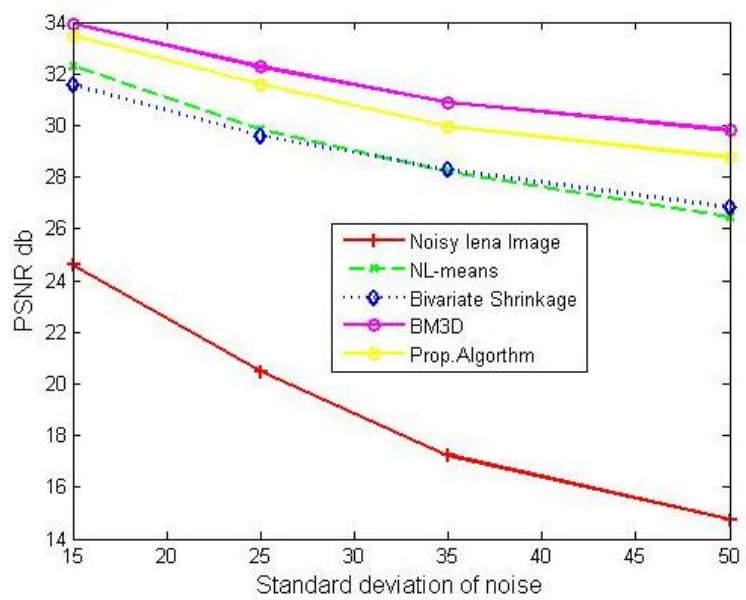

Fig 4(a): PSNR Vs Standard deviation of noise of color Lena Image

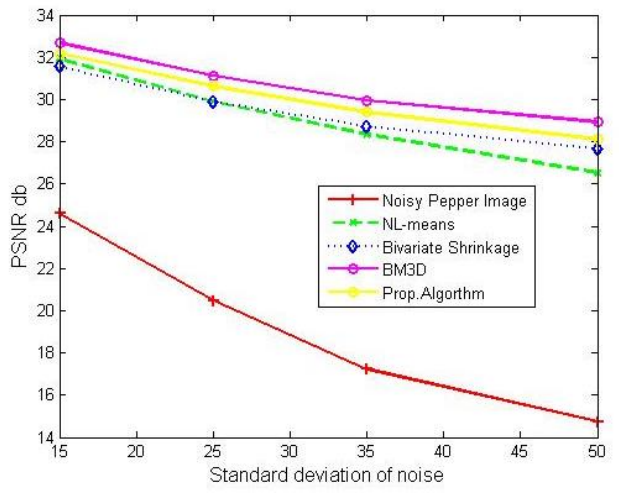

Fig 4(b): PSNR Vs Standard deviation of noise of color Peppers Image

\section{CONCLUSION}

Most available techniques yields good results in terms of PSNR but fail to preserves the edges and fine details of an image. The proposed algorithm addresses this problem to a large extent. The proposed algorithm yields better PSNR for low and moderate standard deviation of zero mean Gaussian noise.

The recursive application of the algorithm is time consuming like any other denoising algorithm however it does not improve PSNR considerably. The proposed edge preservation technique yield good continuity of edges and preservation of fine details along with yielding good PSNR nd SSIM. The proposed algorithm out performs all at very high noise levels in terms of SSIM on all the images. So the spatial domain edge detection and the edge type based conditional mean filtering yields improved edge preservation. The modified Bivariate shrinkage model with the improved threshold also offers improved denoised results in terms of PSNR and SSIM.

It should be noted here that, the proposed algorithm does not require any prior knowledge about the noise properties like standard deviation and is computationally efficient. The numerical results in terms of PSNR and SSIM are much better compared to NL means and the published Bivariate shrinkage for moderate magnitudes $(\sigma=15$ to 35$)$ of noise. For high levels of noise the proposed algorithm does not perform better than BM3D in terms of PSNR. However, it outperforms BM3D in terms of SSIM at high noise magnitudes $(\sigma=$ 80,100 etc). Due to its single pass execution and no requirement of weighted Gaussian averaging, it is computationally efficient compared to BM3D and NL means. NL means introduces too much of blurring at higher noise levels while BM3D introduces contours in the smoothly varying image regions. The proposed algorithm neither blurrs the image nor introduces contours in the smooth regions.

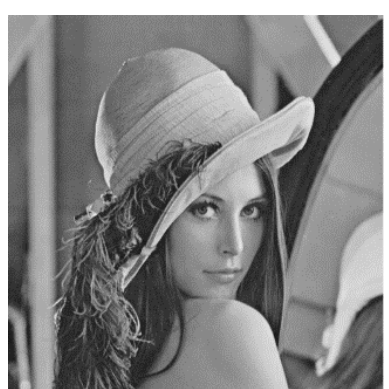

(a)

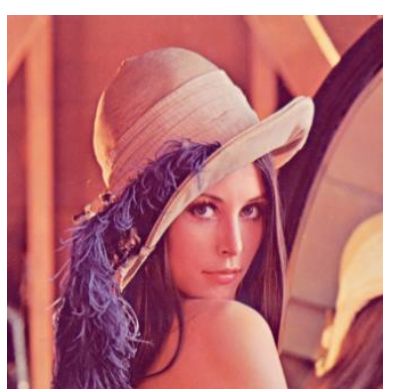

(b)
Fig 5: Noise free Images (a) Lena grey (b) Lena color 

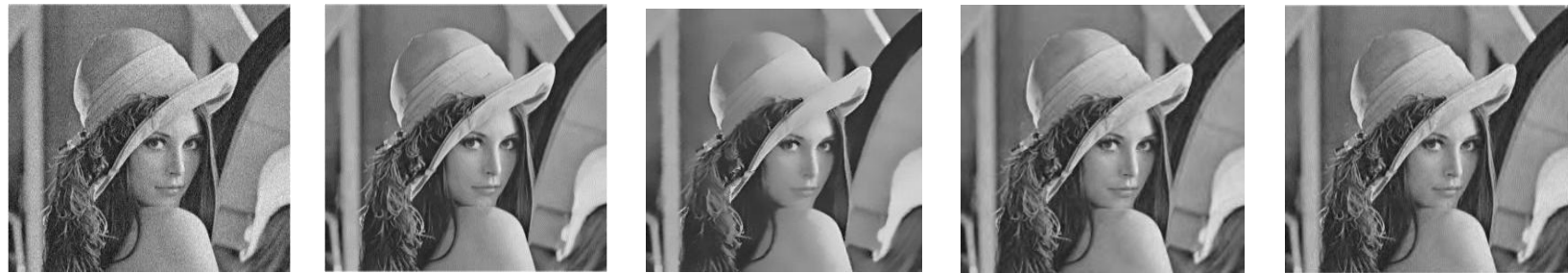

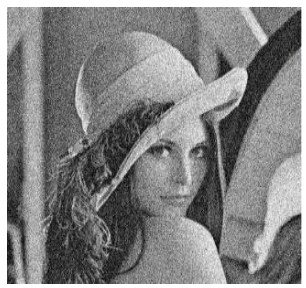

(a)

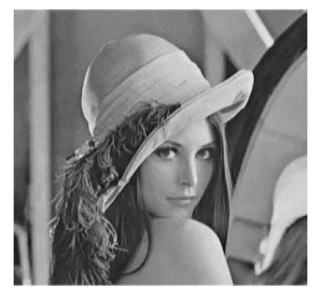

(b)

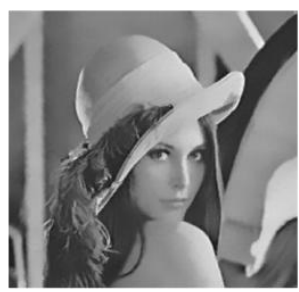

(c)

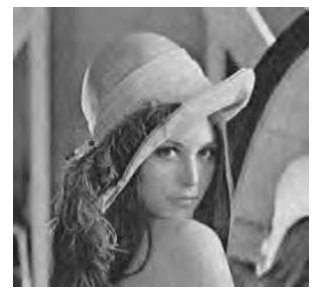

(d)

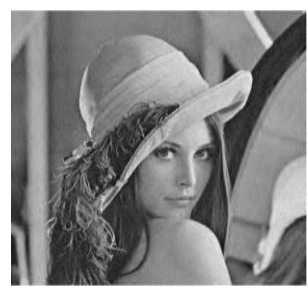

(e)

Fig 6: Noisy Image of Lena (std_dev=15,35) (b) BM3D (c) NL-means d)Bivariate (e) Proposed Algorithm
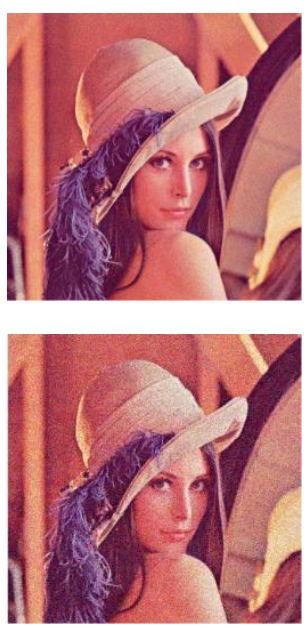

(a)
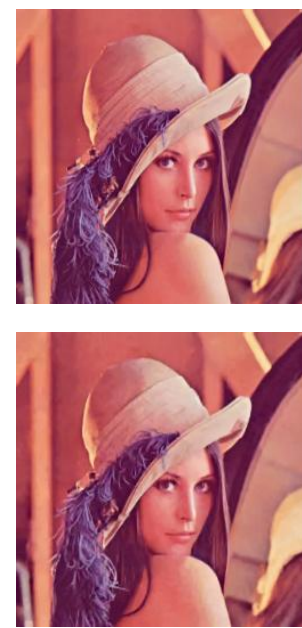

(b)
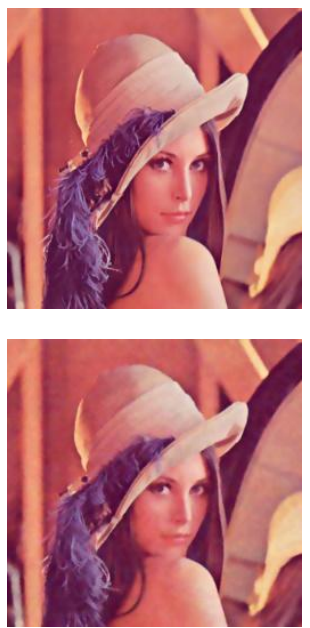

(c)
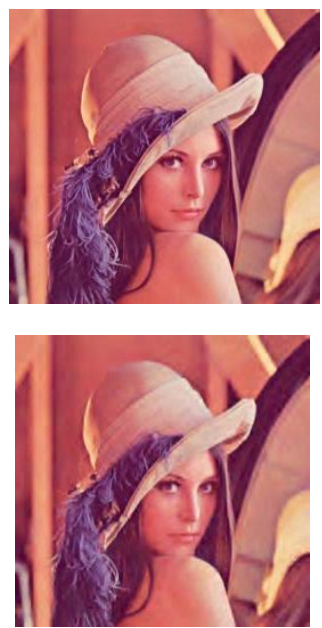

(d)
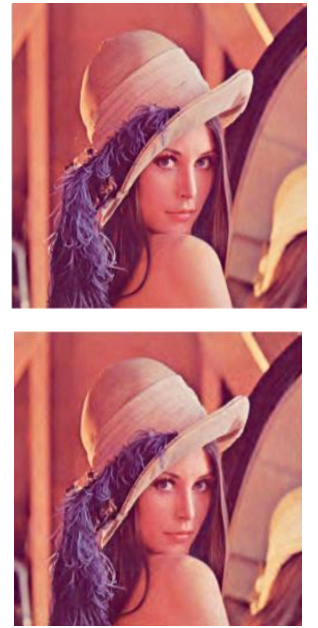

(e)

Fig 7: Noisy Image (std_dev=15,35) (b) BM3D (c) NL-means (d)Bivariate (e) Proposed Algorithm

\section{REFERENCES}

[1] L. Shao, R. Yan, X. Li, and Y. Liu, "From heuristic optimization to dictionary learning: $A$ review and comprehensive comparison of image denoising algorithms," Cybernetics, IEEE Transactions on, vol. 44, no. 7, pp. 1001-1013, July 2014.

[2] K. Dabov, A. Foi, V. Katkovnik, and K. Egiazarian, "Image denoising by sparse 3-d transform-domain collaborative filtering," Image Processing, IEEE Transactions on, vol. 16, no. 8, pp. 2080-2095, Aug 2007

[3] Jin Guoying ,ChenHongye, "Wavelet Local Denoise Reconstruction Algorithm based on non-separable MRA", International Forum on Information Technology and Applications, 2010.

[4] V.NagaPrudhvi Raj and Dr. T Venkateswarlu, " Denoising of Medical Images Using Undecimated Wavelet Transform ", IEEE, pp. 483-488, 2011.

[5] R. R. Coifman \& D. L. Donoho, "Translation invariant denoising in Wavelets and Statistics", Springer-Verlag, Eds. New York, 1995, pp.125-150.
[6] N. G. Kingsbury, "A dual-tree complex wavelet transform: a new efficient tool for image restoration and enhancement," Proc. EUSIPCO'98, Rhodes, Sept. 1998, pp. 319-322.

[7] P.R. Hill, A. Achim, D.R. Bull and M.E. Al-Mualla, "Dual-tree complex wavelet coefficient magnitude modelling using the bivariate Cauchy-Rayleigh distribution for image denoising”, Signal Processing 105, Elsevier, 2014, pp. 464-472.

[8] M. Crouse, R. Nowak, and R. Baraniuk, "Wavelet-based statistical signal processing using hidden Markov models", IEEE Trans. Signal Processing, vol. 46, No.4,pp. 886-902, April 1998.

[9] Semler, L., \& Dettori, L. "A Comparison of WaveletBased and Ridgelet-Based Texture Classification of Tissues in Computed Tomography", Proceedings of International Conference on Computer Vision Theory and Applications, 2006, pp.1-5.

[10] G. Y. Chen, T. D. Bui and A. Krzyzak, "Rotation Invariant Pattern Recognition using Ridgelet, Wavelet Cycle-spinning and Fourier Features," Pattern Recognition vol 38, 2005, pp. 2314- 2322. 
[11] G. Y. Chen and B. Kegl, "Complex Ridgelets for Image Denoising" Department of Computer science and Operations Research, Unversity of Montreal, CP 6128 succ. Centre-Ville, Montreal, Quebec, Canada H3C 317.

[12] G. Y. Chen and B. Kegl. "Image denoising with complex ridgelets," Pattern Recognition, Elsevier, vol. 40, pp. 578-585, 2007.

[13] Rajlaxmi Chouhan, Rajib Kumar Jha and Prabir Kumar Biswas, " Image Denoising using Dynamic Stochastic Resonance in Wavelet domain ", 12th International Conference on Intelligent Systems Design and Applications (ISDA), IEEE, pp. 58-63, 2012.

[14] Baudes A, BartomeuColl, Jean Michel Morel, "Image Denoising by non-Local Averaging" Proceedings of ICASSP 2005, IEEE, 0-7803-8874-7, 2005.

[15] A. Buades, B. Coll, and J. M. Morel, "On Image Denoising Methods," Technical Report 2004-15, CMLA, 2004, pp.1-40

[16] A. Buades, B. Coll, J.M. Morel "A Non local algorithm for image denoising", IEEE Computer Vision and Pattern Recognition, Vol. 2, pp.60-65, 2005.

[17] Neelam Mehala, RatnaDahiya, "A Comparative Study of FFT, STFT and Wavelet Techniques for Induction Machine Fault Diagnostic Analysis", Proc. of the 7th WSEAS Int. Conf. on Computation al Intelligence, ManMachine Systems and Cybernetics (CIMMACS), 2008, pp. 203-208.

[18] David L. Donoho and Iain M. Johnstone. "Adapting to Unknown Smoothness via Wavelet Shrinkage." Journal of the American Statistical Association, Vol. 90, No. 432, pp. 1200-1224, Dec. 1995.

[19] E. P. Simoncelli, "Bayesian denoising of visual images in the wavelet domain," in BayesianInference in Wavelet Based Models, P. Müller and B. Vidakovic, Eds. New York: Springer-Verlag, 1999.

[20] LeventSendur and Ivan W. Selesnick, "Bivariate Shrinkage With Local Variance Estimation", IEEE Signal Processing Letters, Vol. 9, No. 12, December 2002.

[21] E. LePennec and S. Mallat, "Image compression with geometrical wavelets", In IEEE Int. Conf. Image Processing, Vancouver, Canada, September 2000

[22] WangCan, SuWeimin, GuHong, ShaoHua, " Edge Detection of SAR Images using Incorporate ShiftInvariant DWT and Binarization Method ", ICSP2012 Proceedings IEEE, pp. 745-748, 2012.

[23] D. Marr and E. Hildreth, "Theory of edge detection," Proc. Royal Society of London, B 207 pp.187-217, 1980.
[24] G. Chen, T. Bui, and A. Krzyzak, "Image denoising using neighbouring wavelet coefficients," in Acoustics, Speech, and

[25] Signal Processing, 2004. (ICASSP '04). IEEE International Conference on, vol. 2, May 2004, pp. ii917-20 vol.2

[26] L. Sendur and I. Selesnick, "Bivariate shrinkage functions for wavelet-based denoising exploiting interscale dependency," Signal Processing, IEEE Transactions on, vol. 50, no. 11, pp. 2744-2756, Nov. 2002.

[27] L. Sendur and I. Selesnick, "Bivariate shrinkage with local variance estimation," Signal Processing Letters, IEEE, vol. 9, no. 12, pp. 438-441, Dec. 2002.

[28] A. Pizurica, W. Philips, I. Lemahieu, and M. Acheroy, "A joint inter- and intrascale statistical model for Bayesian wavelet based image denoising," IEEE Trans. Image Processing, vol. 11, pp. 545-557, 2002.

[29] "Digital Image Processing," R. C. Gonzales and R. E. Woods, $3^{\text {rd }}$ ed., Pearson Education Inc., 2012, pp. 350404 .

[30] T. D. Bui and G. Y. Chen,"Translation invariant denoising using multiwavelets," IEEE Transactions on Signal Processing, vol.46, no.12, pp.3414-3420, 1998

[31] S. Chang, B. Yu, and M. Vetterli, "Adaptive wavelet thresholding for image denoising and compression," IEEE Trans. Image Processing, vol. 9, pp. 1532-1546, Sept. 2000

[32] S. G. Chang, B. Yu, and M. Vetterli, "Spatially adaptive wavelet thresholding with context modeling for image denoising," IEEE Trans. ImageProcessing, vol. 9, pp. 1522-1531, Sept. 2000.

[33] Firoiu I, Isar A, Isar D , “A Bayesian approach of wavelet based image denoising in a hyperanalytic multiwavelet context". WSEAS Trans Signal Process 6:155$164,2010$.

[34] Yin M, Liu W, Zhao X, Guo Q-W, Bai R-F , “ Image denoising using trivariate prior model in nonsubsampled dual-tree complex contourlet transform domain and nonlocal means filter in spatial domain". Opt Int J Light Electron Opt 124:6896-6904, 2013.

[35] D. L. Donoho and I. M. Johnstone, "Adapting to unknown smoothness via wavelet shrinkage," J. Amer. Stat. Assoc., vol. 90, pp. 1200-1224, 1995.

[36] H. Guo. Theory and Applications of the Shift-Invariant, Time-Varying and Undecimated Wavelet Transforms.Master's Thesis, Dept. ECE, Rice University, Houston, TX, 1995

[37] A. Gyaourova, C.Kamath, IK Fodor, "Undecimated wavelet transforms for image de-noising" November 19 , 2002 